\title{
Emergency toxicology: Timely antidotes and giving poisoned organs a second life - CORRIGENDUM
}

\section{Sophie Gosselin}

doi: 10.1017/cem.2018.491

In the original publication of this article, Dr. Gosselin's affiliation were inaccurate and should read as follows:

Sophie Gosselin, MD CSPQ, FRCPC, FAACT, FACMT

Centre antipoison du Québec, Québec, Canada

Hôpital Charles-Lemoyne, Greenfield Park, Canada

McGill University Health Centre, Montréal, Canada

\section{REFERENCE}

Gosselin S. Emergency toxicology: Timely antidotes and giving poisoned organs a second life. CFEM 2019;21(1): 3-4. doi: 10.1017/cem.2018.491. 